\title{
Fade to Black \\ Das Schwarzbild im Film als Angstbild
}

Florian Leitner

In Martin Heideggers Sein und Zeit wird die Angst zur »Grundbefindlichkeit« erklärt, in der das Dasein »in einem ursprünglichen Sinne erschließbar « wird. ${ }^{1}$ Vor diesem Hintergrund ist es naheliegend, dass ein Journal, das die Hermeneutik im Titel trägt, sich der Angst widmet. Schließlich bildet Sein und Zeit nicht nur den Ausgangspunkt einer modernen Hermeneutik, sondern schreibt in diese die Angst als privilegierten Zugang zum Verstehen des Seins ein, fast so wie Sigmund Freud in die Psychoanalyse den Traum als Königsweg zum Unbewussten einschreibt. Und so wie die Interpretation von Träumen für die Psychoanalytiker das Unbewusste auslegbar macht, können die Philosophen möglicherweise allein aus der denkenden und empfindenden Introspektion zum Verstehen der Angst und daran anschließender Facetten der Existenz gelangen. Medienwissenschaftler legen ihren Untersuchungen hingegen technisch hervorgebrachte Artefakte zugrunde, die außerhalb des menschlichen Denkens und Fühlens liegen. Für uns stellt sich daher die Frage, ob sich derartige Artefakte finden lassen, die zu einer Hermeneutik der Angst beitragen können. Diese müssen bei den Mediennutzern nicht unbedingt tatsächlich Bedrohungsgefühle hervorrufen. Die medienwissenschaftliche Grundannahme besteht in diesem Zusammenhang vielmehr darin, dass eine Struktur wie die Angst, ob man sie nun als Existenzial oder kulturelles Leitkonzept fassen will, nicht nur als menschliche Befindlichkeit auftritt, sondern auch in Gestalt medientechnischer Anordnungen. Anders gesagt stellt aus dieser Perspektive das Erleben von Gefühlen durch den psychischen Apparat von Menschen nur eine von vielen Varianten dar, in denen Angst prozessiert wird. Man kann davon ausgehen, dass es auch andere Apparate gibt, in denen dies geschieht - zunächst einmal ohne dass sich jemand oder etwas identifizieren lässt, der, die oder das Angst spürt.

Ein Vorgehen, das den Angstbegriff in dieser Weise vom menschlichen Erleben ablöst, trägt der Idee einer »Familienähnlichkeit von Angstphänomenen" Rechnung, wie sie der vorliegenden Ausgabe der Hermeneutischen Blätter zugrunde liegt. Das heißt, es verwirft die

${ }_{1}$ Martin Heidegger, Sein und Zeit [1927], Tübingen ${ }^{15} 1984,190, \$ 40$. 
Vorstellung von allgemeinen Merkmalen, die auf alle Angstphänomene gleichermaßen zutreffen. Auch wenn Angst häufig etwas ist, das von Lebewesen verspürt wird, muss das nicht für alle ihre Erscheinungsformen gelten. Sie kann jenseits dessen auch als Dynamik auftreten, die sich in bestimmten medientechnischen Operationen manifestiert. Im Folgenden möchte ich eine bestimme Klasse solcher Operationen herausgreifen und argumentieren, dass das von ihnen hervorgebrachte Artefakt als Angstphänomen begriffen werden kann. Das Artefakt, von dem die Rede ist, kann man als "Schwarzbild« bezeichnen. Dem Schwarzbild als Angstbild möchte ich mich in fünf Schritten nähern: Nach einigen kurzen Bemerkungen zur Schwärze im Film anhand des Beispiels Nosferatu (1.) soll dargelegt werden, wie das filmische Schwarzbild einerseits auf das Medium selbst verweist (2.), dabei andererseits aber stets zwischen Abstraktion und filmischer Repräsentation oszilliert (3.). Ausgehend vom zweiten Filmbeispiel Leviathan (4.) will ich schließlich auf Grundlage der Unterscheidung von Angst und Furcht die Kernstruktur des Schwarzbilds als Intentionalität ohne Gegenstand akzentuieren, wie sie auch für andere Angstphänomene benannt worden ist (5.).

\section{Black Screens of Death}

Mit die eindringlichste visuelle Gestalt, die man als Abwandlung des Schwarzbilds sehen kann, sind gegenwärtig die Hinweis-Bildschirme, die verschiedene Computer-Betriebssysteme anzeigen, wenn nichts mehr geht. Der komplett schwarze Hintergrund wird dabei lediglich durch ein paar weiße Buchstaben unterbrochen, die darüber informieren, dass ein kritischer Fehler aufgetreten ist und das System neu gestartet werden muss. In der Computerkultur spricht man in diesem Zusammenhang vom "black screen of death" und deutet damit an, dass in digitalen Zeiten kaum etwas angstbesetzter ist als ein Schwarzbild, das von einem Systemcrash zeugt. An dieser Stelle soll es allerdings um eine Form des Schwarzbilds gehen, die nicht als Information über den internen Zustand einer havarierten Maschine dient, sondern als ästhetisches Phänomen fungiert: das Schwarzbild im Film. 


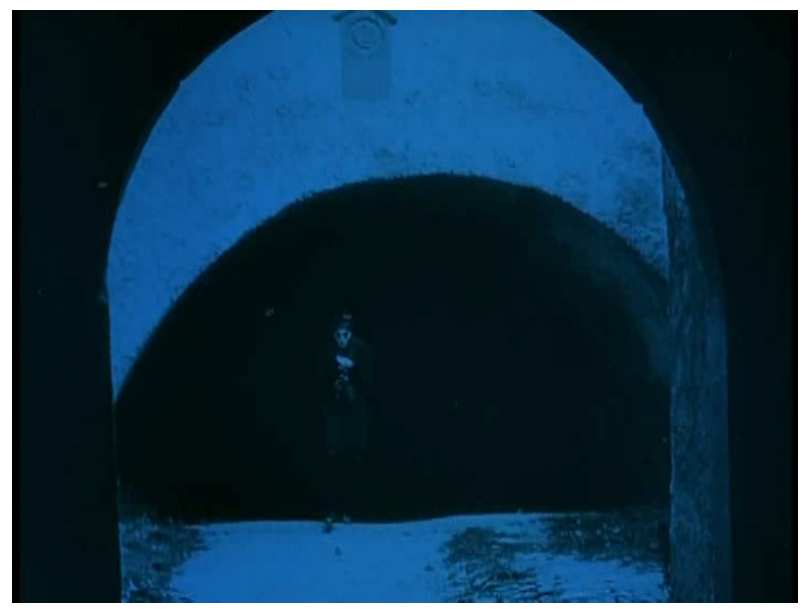

Nosferatu (1922)..$^{2}$

Es liegt auf der Hand, dass Schwärze im Film ganz allgemein nicht zuletzt dort eine herausgehobene Rolle spielt, wo es um Angstmotive geht, und dass sie unter anderem in Verbindung mit der Ästhetik des Horrors steht. Ein Beispiel aus der Frühgeschichte des Genres findet sich in Friedrich Wilhelm Murnaus Nosferatu aus dem Jahr 1922. Am Ende des ersten Akts kommt der männliche Protagonist Hutter beim Grafen Orlok an und wird von diesem in seinem Schloss empfangen. Der Übergang von der Welt des Gewöhnlichen in die Welt des Unheimlichen und Bösen wird hier vollzogen, indem Hutter durch drei Torbögen schreitet, die ihn in das Innere des Gebäudes führen. Bereits nachdem er den ersten durchquert hat, deutet sich an, dass es hier nicht mit rechten Dingen zugeht: Das Tor schließt sich hinter ihm wie von Geisterhand, so dass Hutter, als er sich umdreht und verunsichert zurückblickt, auf eine schwarze Fläche im Zentrum der Einstellung starrt. Vor allem aber das dritte Tor wird von der expressionistischen Kamera so präsentiert, dass sich das filmische Bild in einer Komposition monochromer geometrischer Formen auflöst. Wieder steht die gähnende schwarze Fläche unter dem Torbogen im Zentrum, aus welcher der gespenstische Orlok hervorgetreten ist, als ob er sich aus dem Nichts heraus materialisiert hätte, um seinen Besucher zu empfangen. Der verschwindet dann

2 Bei der Abbildung handelt es sich um einen Screenshot zur Zitation, der vom Autor von folgender Quelle angefertigt worden ist: Nosferatu. Eine Symphonie des Grauens, Kauf-DVD: Leonine 2014. 
am Ende der Szene zusammen mit dem Vampir in diesem Schwarz, das damit zum Urgrund wird, dem das Böse entspringt und in den es sein Opfer hinabzieht. Für Hutter wird die schwarze Fläche, die

vor uns Zuschauern auf der Kinoleinwand erscheint, zum wortwörtlichen »black screen of death«, zum Menetekel eines Todes, dem er selbst zwar noch entfliehen kann, dem am Ende aber seine Gattin, nachdem sie von Orlok gebissen worden ist, zum Opfer fallen wird.

\section{Das Medium im Leerlauf}

Unabhängig von einer symbolhaften Interpretation kann man die schwarzen Flächen im Filmbild von Nosferatu natürlich auch einfach als das betrachten, was sie innerhalb der fiktionalen Welt repräsentieren: Areale der Dunkelheit. Murnaus Film fügt sich damit in eine Traditionslinie der Horrorästhetik ein, deren theoretische Beschreibung auf Edmund Burkes Abhandlung über das Erhabene und Schöne aus dem Jahr 1757 zurückgeführt werden kann. Dunkelheit stellt für Burke eine der Drohungen dar, die jenen erhabenen Schrecken hervorrufen können, der etwa in der Kunst zum "delightful horror" und damit zur Quelle ästhetischen Vergnügens werden kann. Schwärze wiederum sei nichts anderes als partielle Dunkelheit, erklärt Burke. ${ }^{3}$ Vor diesem Hintergrund gibt es noch eine weitere Möglichkeit, wie die schwarzen Flächen in Nosferatu gelesen werden können: nicht nur als Darstellung düsterer Winkel in Orloks Schloss oder als Urgrund des Bösen, sondern auch als Verweis auf die Dunkelheit am Anfang des Kinos.

"Am Anfang ist das Dunkel der Projektion. ${ }^{4}$ Mit diesen Worten leitet Ute Holl eines der Kapitel ihrer Studie Kino, Trance und Kybernetik ein. Die Dunkelheit steht nicht nur am Beginn jeder Kinovorführung, bevor der Apparat anläuft, um Lichtbilder zu projizieren. Das Dunkle, Obskure, das in der quasi hypnotischen Trance mitschwingt, in die Filme ihr Publikum versetzen, bildet auch einen Ausgangspunkt der Geschichte der Filmtheorie. Sie ist eine Geschichte des Nachdenkens über einen psychischen Zustand, in dem Menschen Dinge sehen, die nicht da sind, und Bewegung wahrnehmen, wo in Wirklichkeit nur unbewegte Einzelbilder aufeinander folgen. Und schließlich ist die Dunkelheit auch während des Films fortwährend präsent, wie Holl ausführt: »Weil die Flügel-

3 Edmund Burke, A Philosophical Enquiry into the Origin of our Ideas of the Sublime and Beautiful, London 1757, 145.

4 Ute Holl, Kino, Trance und Kybernetik, Berlin 2002, 37. 
blende, während das Malteserkreuz die Einzelbilder intermittierend transportiert, den Projektionsstrahl im entsprechenden Rhythmus stroboskopiert, sitzen Kinozuschauer fast die Hälfte der Projektionszeit im Dunkeln, während ihre Sehnerven im Takt dieser Mechanik gereizt werden. $\aleph^{5}$

Ohne es zu merken, blickt das Kinopublikum die halbe Zeit auf eine schwarze Leinwand. Begreift man letztere als Schwarzbild, so werden im Kino während der einen Sekunde, in der die 24 Kader eines Filmstreifens durch den Projektor laufen, mindestens 96 Bilder gezeigt: Aus wahrnehmungsphysiologischen Gründen wird jedes filmische Einzelbild mindestens zweimal projiziert, unterbrochen durch ein Schwarzbild. Dieses ist nicht auf dem Filmstreifen enthalten, erscheint aber auf der Leinwand, wenn die Umlaufblende den Lichtstrahl unterbricht. Ein weiteres Schwarzbild erscheint, wenn der Lichtstrahl noch mal unterbrochen wird, um den Filmstreifen zum nächsten Kader zu transportieren. Auch in der digitalen Technologie wird die »black frame insertion « gelegentlich verwendet, um Bewegungsunschärfen zu reduzieren. Für das analoge Kino gilt indes ausnahmslos: Mindestens jedes zweite Bild, das auf der Leinwand erscheint, ist ein Schwarzbild. Wir sehen diese schwarzen Bilder nur nicht.

Selbstverständlich gibt es im Film auch jene anderen schwarzen Bilder, die wir sehr wohl sehen. Zum Beispiel blicken wir in Spielfilmen häufig dann auf eine schwarze Leinwand, wenn sich narrative Sprünge oder Brüche ereignen, oft in Verbindung mit Auf- und Abblenden. »Fade to black«, steht dann im Drehbuch. Als technical term steht der Begriff "Schwarzbild « in Zusammenhang mit diesen sichtbaren schwarzen Bildern und bezeichnet den einzelnen unbelichteten Filmkader, der auch bei geöffneter Umlaufblende das Licht des Projektors nicht durchlässt. Ein distinktes schwarzes Bild auf der Leinwand erscheint natürlich erst dann, wenn auf dem Filmstreifen eine Reihe unbelichteter Kader aufeinander folgen, was dann als "Schwarzfilm» bezeichnet wird. Wie bereits deutlich geworden sein dürfte, verwende ich den Ausdruck "Schwarzbild" in einem Sinn, der über diese rein technische Beschreibung hinausgeht. Dass ich dabei auf einen Fachbegriff aus der Schnitt- und Projektionspraxis zurückgreife, erklärt sich auch aus der Eigenheit der diesem zugrunde liegenden Wortbildung: Rein grammatikalisch gesehen ist das Schwarzbild nicht einfach nur ein schwarzes Bild, sondern ein Bild von Schwarz, ein Bild, das Schwarz zum Bildinhalt hat. Es ist ein abstraktes Bild.

5 Ebd. 
Als das schwarze Rechteck, das es ist, erinnert das filmische Schwarzbild an Kasimir Malewitschs Schwarzes Quadrat, ein Urbild visueller Abstraktion. Es ist abstrakt in dem Sinne, dass es nichts repräsentiert. Nichts ist in ihm zu sehen, das sich auf ein Außerhalb des Bildes bezieht. Selbstverständlich gibt es das erwähnte Schwarzbild im Spielfilm, das etwa für zeitliche Sprünge in der Handlung steht, doch dieser Sinnbezug ergibt sich aus dem Erzählfluss, den das Schwarzbild unterbricht, aus seinem Kontext - so wie der Bezug zu religiösen Bildpraktiken nicht durch Malewitschs Gemälde als solchem hergestellt wird, sondern durch den Ausstellungskontext bei seiner ersten Präsentation im Jahr 1915 in Sankt Petersburg, bei der das Gemälde in einer Ecke des Raums hing, am höchsten Punkt, leicht nach unten geneigt, wie eine Ikone.

Im Gegensatz zum Schwarzbild des Films tritt uns Malewitschs Quadrat nicht als vollständig schwarze Leinwand entgegen, sondern als schwarzes Viereck auf weißem Grund. So ist dann auch das passendere malerische Äquivalent zum filmischen Schwarzbild nicht Malewitschs schwarzes, sondern sein Weißes Quadrat aus dem Jahr 1919: ein weißes Rechteck auf einem ebenfalls weißen, aber in einem anderen Ton gehaltenen Grund. Es erinnert an die Leinwand des Malers in ihrem rohen, unbearbeiteten Zustand, bevor sich darauf ein Bild eingeschrieben hat. Damit entspricht es der schwarzen Leinwand im Kino, der medialen Oberfläche im Ausgangszustand, als das Licht bereits verlöscht, aber der Film noch nicht angelaufen ist. Es bezeichnet den unmarked state des Mediums, bevor die medialen Zeichen darauf erscheinen - das leerlaufende Medium, das Medium ohne Botschaft, in einem Zustand, für den noch mehr als sonst Marshall McLuhans Diktum gilt: "The medium is the message." Wenn das Schwarzbild eine Botschaft hat, dann ist das Medium seine Botschaft. Man kann auch sagen, das Schwarzbild als abstraktes Bild ist ein Bild der Erstmaterie des filmischen Mediums. So argumentiert Richard Misek in seinem Videoessay The Black Screen: "Leibniz referred to the underlying element of all substances as primary matter. For him, primary matter is what gives the universe its unity. Cinema's primary matter is black. $\ll^{6}$

\footnotetext{
6 Richard Misek, The Black Screen, in: Journal of Videographic Film \& Moving Image Studies 4.2a (2017), http://mediacommons.org/intransition/2017/06/01/ black-screen (28.01.2020).
} 


\section{Die doppelte Referenz des Schwarzbilds}

Wenn uns in den weißen Leinwänden der Malerei und den schwarzen Leinwänden des Films das Medium in seinem unmarked state vor Augen tritt, dann könnte umgekehrt gesagt werden, dass im schwarzen Rechteck das Medium der Malerei im überdeterminierten $\mathrm{Zu}-$ stand erscheint. Denn man kann Malewitschs Schwarzes Quadrat auch als Endresultat einer Prozedur interpretieren, bei der alle möglichen Zeichen der Malerei übereinandergelegt wurden - so wie es in Honoré de Balzacs Erzählung Le Chef-d'euvre inconnu/Das unbekannte Meisterwerk der Altmeister Frenhofer tut, der an dem titelgebenden Gemälde zehn Jahre arbeitet, Farbschicht um Farbschicht hinzufügt, bis irgendwann nichts mehr zu erkennen ist. Im Gegensatz dazu generiert das kinematografische Medium im überdeterminierten Zustand, zumindest wenn man es mit der Fotografie verschaltet, weiße Rechtecke, wie man sie in den Fotos der Serie Theatres von Hiroshi Sugimoto aus dem Jahr 1978 sieht. ${ }^{7}$ Sugimoto hat seine Fotokamera in alten amerikanischen Lichtspielhäusern aufgebaut und eine extrem lange Belichtungszeit von etwa zwei Stunden gewählt, während der jeweils ein kompletter Film projiziert wurde. Auf den Fotos ist an der Stelle der Projektionsleinwand ein weißes Rechteck zu sehen - das Ergebnis der Überlagerung aller einzelnen Kader eines Films, das, was herauskommt, wenn man einen Film von Anfang bis Ende fotografiert. Das Foto eines Films ist ein weißes Rechteck. Und so strahlend schön und jungfräulich dieses Weiß auf Sugimotos Fotos scheinen mag, es ist nicht das Bild eines Mediums im Zustand des unmarked state, das unendliche Möglichkeiten der Zeichengenerierung bietet, sondern das Bild eines Mediums, das sich durch die Addition seiner unzähligen Möglichkeiten verschlossen hat, auf dessen Oberfläche nichts mehr zur Erscheinung gebracht werden kann. Das Schwarzbild hingegen ist maximal offen, es gibt nichts filmisch Vorstellbares, für dessen Erscheinung es nicht zum Ausgangspunkt werden könnte. Es beinhaltet damit auch ein cineastisches Glücksversprechen.

Diesem Glücksversprechen steht das Unheimliche, Bedrohliche gegenüber, das mit der Schwärze assoziiert ist. Wenn der Film dieses Unheimliche ausspielt, dann fällt es in gewisser Weise auf sein eigenes Medium zurück. So kann das partielle Schwarzbild in Nosferatu wie gesagt unter anderem gelesen werden als Repräsentation eines $\mathrm{Ab}-$ schnitts des fiktionalen Raums, aus dem derVampir hervortritt, aber eben auch als Verweis auf die Medialität des Films. Es wird durch

\footnotetext{
7 http://www.sugimotohiroshi.com/new-page-7 (17.02.2020).
} 
eine bläulich-weiße Mauer umfasst, die wiederum von einer weiteren schwarzen Fläche umschlossen wird, wodurch die Rahmung der Kinoleinwand innerhalb des Filmbildes reproduziert wird - wenn auch nicht geometrisch korrekt, da das partielle Schwarzbild, das den dunklen Torbogen darstellt, kein Rechteck ist. Trotzdem wird es, spätestens wenn Orlok völlig unvermittelt in ihm auftaucht, zum übernatürlichen Pendant der Kinoleinwand, auf der wie aus dem Nichts künstliche aber lebensecht wirkende Gestalten erscheinen. Dem partiellen Schwarzbild in Nosferatu kann somit eine doppelte Referenz zugesprochen werden: Es verweist auf etwas innerhalb der fiktiven Welt des Films, auf einen düsteren Winkel in Orloks Schloss, aber auch auf das Medium, in dem diese Welt zur Erscheinung gebracht wird, und das den Zuschauern dadurch ebenfalls unheimlich werden kann.

Eine solche doppelte Referenz kennzeichnet nicht nur das partielle, sondern auch das vollständige Schwarzbild, gerade auch im Horrorfilm. Zahlreich sind dort die Szenen, in denen die Kamera in einen düsteren Bereich der fiktionalen Welt eindringt, oft in einen Keller oder eine Grabstätte, und das Bild wird dann zumindest für einige Sekunden komplett schwarz. In solchen Fällen ist das Schwarzbild zunächst einmal ein repräsentationales Bild, das uns die Dunkelheit im diegetischen Raum zeigt. Es bleibt aber zugleich auch ein abstraktes Bild, das auf sein Medium verweist. Am deutlichsten wird das, wenn man den Film in einem Kino oder einem anderen abgedunkelten Raum sieht: Man wird dann nicht nur durch die Unheimlichkeit der Dunkelheit des fiktionalen Raums affiziert, den die Kamera zusammen mit den Filmfiguren betritt und in dem mutmaßlich gefährliche Kreaturen lauern; man findet sich darüber hinaus auch selbst in einem dunklen Raum wieder, in dem die einzig verbliebene Lichtquelle, die Leinwand oder der Bildschirm, nicht mehr scheint und der so zum Nicht-Raum wird. "When the screen goes black, my room disappears", heißt es in Miseks Videoessay. Für einen Moment werden nicht nur die fiktionalen Figuren, sondern auch die Zuschauer in eine potentiell unheimliche Situation gebracht - durch das Schwarzbild, das als repräsentationales Bild die Dunkelheit im diegetischen Raum repräsentiert, als abstraktes Bild aber einfach auf der medialen Oberfläche die Manifestation des Leerlaufs des darunterliegenden Mediums ist. Wie beim black screen of death auf dem Computermonitor hat auch dieses Schwarzbild mit der Unheimlichkeit eines Mediums zu tun, das sein Kerngeschäft - welches beim Film zunächst einmal darin besteht, Licht auf eine 
beziehungsweise von einer Fläche scheinen zu lassen - kurzzeitig ausgesetzt hat.

\section{Leviathan und Moby-Dick}

In dem Potential des Schwarzbilds, abstraktes und repräsentationales Bild zugleich zu sein, realisiert sich das "Spiel von materialer Gegenständlichkeit und unaufhörlicher Semiotisierung«, das der Film für Gertrud Koch "mit uns treibt « - wodurch er uns "das Verhältnis selbst vor Augen « stellt, »das wir als denkende und sehende Wesen zur Welt einnehmen«. Als mustergültige Manifestation dieser Dynamik nennt Koch dann auch den Schwarzfilm, »der mal als unbestimmter narrativer Raum, der alle und keinen Raum enthalten kann, mal als monochrome Farbfläche erfahren werden kann «. ${ }^{8}$

Eines der bemerkenswertesten Beispiele für diese Dialektik aus den vergangenen Jahren findet sich am Anfang des auch ansonsten bemerkenswerten Leviathan von Lucien Castaing-Taylor und Verena Paravel aus dem Jahr 2012. Es dauert mehrere Minuten, bis sich in dem Schwarzbild, mit dem der Film anhebt, und den schemenhaften Umrissen, die sich zaghaft darauf einzuschreiben beginnen, etwas zu erkennen ist. Erst nach und nach werden die zunächst abstrakt wirkenden optischen Gestalten zum Mittel filmischer Repräsentation und geben preis, wo sich die Kamera befindet: auf einem Fischkutter auf hoher See, nachts, als gerade die Netze eingeholt werden. Vollständig klar wird das erst, als die unscharfen visuellen Formen, die anfangs nur in kleinen Teilbereichen des Schwarzbilds immer wieder von Neuem erscheinen und verschwinden, das Schwarzbild schließlich vollkommen überdecken und als Schiffsketten, Kräne, Seemänner in Regenmänteln und dergleichen erkennbar werden.

Der Gegensatz von Abstraktion und Repräsentation wird während des gesamten Films präsent bleiben und am Ende, nach dem Abspann, ein letztes Mal wiederkehren als Schwarzbild, das lange ausgekostet und lediglich von einigen Möwen überlagert wird, die darin als weiße Punkte herumschwirren. Am Anfang von Leviathan wird diese visuelle Dialektik noch dadurch unterstrichen, dass sie sich deutlich von der Tonspur abhebt. Denn die akustischen Zeichen, mit denen der Film beginnt, können relativ schnell zugeordnet werden: das Rauschen der Wellen und des Windes, der metallisch klirrende Lärm der Maschinen an Deck des Schiffs. Das Bild indes kippt

8 Gertrud Koch, Die Wiederkehr der Illusion. Der Film und die Kunst der Gegenwart, Frankfurt a.M. 2016, 74. 
auch im weiteren Verlauf der 20-minütigen Anfangssequenz immer wieder mal kurzzeitig ins Schwarzbild zurück. Dies wird auch für unsichtbare Schnitte genutzt, die dazu beitragen, die hypnotische Qualität des Films zu etablieren, der den Anschein erweckt, einen fortlaufenden Bewusstseinsstrom zu zeigen. Es ist der Bewusstseinsstrom eines Hybridwesens, zu dem der Fischkutter mit seinen Seilwinden, Ketten und Kränen und die menschliche Besatzung verschmelzen - und das durch den Titel und das einleitende Zitat aus dem Buch Hiob mit einem alttestamentarischen Seeungeheuer identifiziert wird: dem Leviathan. In Paravels und Castaing-Taylors Film wird dieser technomythologisch umgedeutet. Hier ist er eine Kreatur, die der industrielle Fischfang gebiert. Sie schwimmt durch den Ozean, sie atmet: Motoren werden angeworfen und pusten Abgase in die Luft. Sie verschlingt die Meeresbewohner: Netze voller Fische werden eingeholt. Sie verdaut: die Fische werden an Deck zerlegt. Sie scheidet aus: Fischabfälle und blutrotes Abwasser fließen zurück ins Meer.

Das Schiff, auf dem Leviathan gedreht wurde, operiert von New Bedford in Massachusetts aus, an dem auch die Handlung von Herman Melvilles Moby-Dick beginnt. Am Anfang des Romans kommt der Erzähler Ishmael in der Hafenstadt an und macht sich auf die Suche nach einer Unterkunft. Es ist bereits Nacht, und so wie die erste Einstellung von Leviathan das Schwarzbild ist, finden sich auf den ersten Seiten von Moby-Dick immer wieder Beschreibungen von Dunkelheit und Schwärze:Von »blocks of blackness, not houses" sieht Ishmael sich umgeben, während er durch die düsteren Straßen wandert, "and here and there a candle, like a candle moving about in a tomb «; $; 9$ als er in einen afroamerikanischen Gottesdienst gerät, blickt er in "a hundred black faces", wie er in einer von rassistischen Untertönen nicht freien Passage berichtet, während der Prediger über "the blackness of darkness" spricht $;{ }^{10}$ und die ausführliche Beschreibung des Spouter-Inn, jener heruntergekommenen Herberge, in der Ishmael sich schließlich einquartiert, hebt am Anfang des dritten Kapitels an mit der über eine Seite sich hinziehenden Beschreibung eines übergroßen Ölgemäldes, das neben dem Eingang des schäbigen Etablissements hängt und das "so thoroughly besmoked, and every way defaced « ist, ${ }^{11}$ dass sich sein Motiv erst nach längerer und wiederholter Betrachtung erschließt. Das Gemälde ist im Lauf der Jahre so stark gedunkelt, das in ihm erst nach und nach einzelne

9 Hermann Melville, Moby-Dick; or, The Whale [1851], Oxford/New York 1988, 8.

${ }^{10}$ Ebd.

${ }^{11}$ Ebd., 10. 
Objekte auszumachen sind, ähnlich wie im Film Leviathan erst nach und nach visuelle Formen im anfänglichen Schwarzbild auftauchen. Doch selbst dann bleibt Schwärze sein zentraler Gegenstand: Was Ishmael fesselt ist "a [...] black mass of something hovering in the centre of the picture". Und so wie die Zuschauer am Anfang von Leviathan die zunächst abstrakt scheinenden Bildelemente erst nach einiger Zeit als etwas entschlüsseln können, das auf ein Außerhalb des Bildes verweist, kann auch Ishmael die schwarze Masse erst allmählich mit einer Referenz versehen: "But stop; does it not bear a faint resemblance to a gigantic fish? even the great leviathan himself? ${ }^{12}$ Der schwarze Fleck stellt einen riesigen Wal dar, gejagt von einem Walfängerschiff. Das Tier wird vom Erzähler mit dem Leviathan aus dem Alten Testament identifiziert, ebenso wie der Pottwal, dem Ishmael in der Crew des Kapitän Ahab hinterherjagen wird. Im Spouter-Inn wird das Ungetüm für ihn erst nach langem Betrachten des Gemäldes sichtbar. Auch der Leviathan des Films, das Hybridwesen aus industriellem Fischkutter und Besatzung, schält sich erst nach längerem Winden aus dem Schwarzbild heraus. Dabei kehrt der Film die Perspektive des Romans auf bemerkenswerte Weise um: Bei Melville ist der Wal das Ungeheuer, das die gesamte Crew des Walfangschiffs bis auf den Erzähler zu Tode bringt; bei Castaing-Taylor und Paravel sind Schiff und Besatzung das Monster.

\section{Angst und Furcht}

Mit Hans Blumenberg kann man den Leviathan als eine Figur des Anderen begreifen: Ihre anthropologische Funktion besteht darin, in der Fiktion des Mythos an die Stelle einer realen Totalität zu treten, mit der die menschliche Kultur etwa in Form der Naturgewalten konfrontiert ist. In diesem Fall ist die Urgewalt der Meere das Andere, welches durch den Anderen, den Leviathan adressierbar gemacht beziehungsweise, wie Blumenberg formuliert, "interpretiert«, "erschlossen«, "besetzt» wird. ${ }^{13}$ Er knüpft hier an Søren Kierkegaards Unterscheidung zwischen gegenstandsloser Angst und objektbezogener Furcht an, die für den modernen Angstbegriff so grundlegend ist: ${ }^{14}$ Dort, wo der Mensch der rohen Natur ausgesetzt ist - in unserem Beispiel der Bedrohung durch den Ozean -, erlebt er eine Situation der Angst; die Gefahr, mit der er sich konfrontiert

\footnotetext{
${ }^{12}$ Ebd., 11.

${ }^{13}$ Hans Blumenberg, Arbeit am Mythos, Frankfurt a.M. ${ }^{21981, ~ 28-29 . ~}$

${ }^{14}$ Søren Kierkegaard, Der Begriff Angst [1844], Hamburg 1984.
} 
sieht, ist objektlos, insofern ihre Ausdehnung unüberschaubar, ihr Ursprung unbekannt, ihre Funktionsweise unverständlich, ihr Sinn rätselhaft bleibt. Die Erfindung des Leviathans als einer Figur, auf die diese Bedrohung projiziert werden kann, löst die Angstsituation in einem Furchtszenario auf. Man kann sie mit Blumenberg als "Kunstgriff" verstehen, der eine "Erklärung für das Unerklärliche«, eine "Benennung für das Unnennbare " liefert und auf diese Weise Angst zu Furcht »rationalisiert . $^{15}$

Sowohl Moby-Dick als auch Leviathan präsentieren zunächst einmal Furchtszenarien. Sie erzählen keineswegs von ungegenständlichen Bedrohungen - Melvilles Wal und Paravels und Castaing-Taylors Fischkutter sind klar definierte Furchtobjekte. Der Ursprung der Gefahr kann benannt werden, doch indem "Leviathan «als mythologischer Signifikant eingeführt wird, wird sie überhöht und zu einem Angstphänomen umcodiert: Der Wal, der riesig genug ist, um ganze Schiffsbesatzungen ins Verderben zu reißen, ist nicht mehr einfach nur ein Meeressäugetier, sondern die monströse Manifestation einer dunklen Macht; der industrialisierte Fischfang, der die Bewohner des Ozeans verschlingt, wird zum die Weltmeere umspannenden Pandämonium. Was zunächst einfach nur die - in der sicheren Distanz der literarischen beziehungsweise filmischen Fiktion erlebte - Furcht vor einer klar umgrenzten Bedrohung darstellt, wird zur unheimlichen Angst vor etwas, das rätselhaft und obskur bleibt. Die Rolle, die das Schwarzbild bei dieser Ausweitung von Furcht zu Angst spielt, erlaubt es, das Schwarzbild als Angstbild zu begreifen.

Die Dynamik von Furcht und Angst wohnt Horrormotiven im Film ganz allgemein inne. In einer Untersuchung über die von Bildmedien hervorgerufenen Bedrohungsaffekte und ihrer Verarbeitung im Film habe ich versucht zu zeigen, dass der schwerer zu fassende Pol dieser Dynamik, das heißt die Angst, dabei als "mediale Angst" lesbar wird: ein Unheimliches, das mit dem Medium selbst in Verbindung steht. Kurz gesagt wird Furcht im Horrorfilm durch das Monster hervorgerufen, Angst durch das Medium, in dem es zur Erscheinung kommt. ${ }^{16}$ Selbstverständlich sind Furcht und Angst dabei nicht, wie etwa letztere bei Kierkegaard, als eine existentielle, die condition humaine überschattende, sondern als ästhetische Erfahrung zu begreifen, die gesucht und genossen werden kann. Das Begriffspaar "Angst" und "Furcht" ermöglicht es, innerhalb dieser Erfahrung zwei Ebenen analytisch voneinander zu unterscheiden,

\footnotetext{
15 Blumenberg, Arbeit am Mythos, 11.

${ }^{16}$ Florian Leitner, Medienhorror. Mediale Angst im Film, Paderborn 2017, 40. Für weitere Beispiele zu Schwarzbildern im Film siehe dort 42-43 und 90-92.
} 
die realiter verwoben sind - ebenso wie sich beim Schwarzbild eine repräsentationale Seite, die etwa das Unheimliche eines dunklen Raums in der Filmrealität evoziert, unterscheiden lässt von einer abstrakten Seite, durch die das Unheimliche eines leerlaufenden Mediums ins Spiel kommt. Die Hypothese, auf welche die vorangegangenen Überlegungen hinauslaufen, lautet, dass durch die Gegenstandslosigkeit, die die abstrakte Seite des Schwarzbilds auszeichnet, die eingangs beschworene Familienähnlichkeit des Schwarzbilds zu Angstphänomenen hergestellt wird:Wenn Blumenberg die Angst als "Intentionalität des Bewusstseins ohne Gegenstand «17 versteht, dann ist sie strukturanalog zum Schwarzbild als abstraktem Bild, das als Intentionalität des Mediums ohne Gegenstand beschrieben werden kann. Nicht umsonst spricht Malewitsch im Zusammenhang seines Schwarzen Quadrats von einer "gegenstandslosen Empfindung" und hält fest: "Es war dies kein ’leeres Quadrat‘, was ich ausgestellt hatte, sondern die Empfindung der Gegenstandslosigkeit. ${ }^{18}$

Um tatsächlich das Schwarzbild selbst als Angstphänomen zu begreifen, darf die Intentionalität ohne Gegenstand nicht wie bei Blumenberg als Bewusstseinsprozess aufgefasst werden, sondern muss als Aspekt einer optomechanischen (oder -digitalen), medientechnischen Operation verstanden werden, der gleichzeitig eine Form des Denkens konstituiert. Man könnte dieses als ästhetisches Denken konzeptualisieren, so wie Dieter Mersch es beschreibt - der sein Argument ausgerechnet am Beispiel Malewitschs illustriert:

"Was ist ein Bild? Handelt es sich notwendig um eine Abbildung, um die Darstellung einer Figur oder Szene, oder lediglich um ein gerahmtes Ding, das alles Mögliche sichtbar machen kann, sogar die Nichtigkeit eines schwarzen Grundes? Man wende sich in diesem Fall an Kasimir Malewitschs Schwarzes Quadrat auf weißem Grund [...]: Es ist ein Bild und doch lässt es im Unbestimmten, ob es ein schwarzes Quadrat auf weißem Grund oder einen weißen Rahmen auf schwarzem Grund zeigt. ${ }^{19}$

Die beiden unterschiedlichen Lesarten sind nicht vereinbar, und so kommt das Gemälde einem nie stillzustellenden Prozess des Denkens gleich, einer unabschließbaren "De-Synthese«, die sich nicht auf ein letztgültiges Urteil festklopfen lässt, welches mit den Kategorien wahr oder falsch bewertet werden könnte. Das Schwarze Quadrat bildet für Mersch die »Synthesis" dieser De-Synthese, und

\footnotetext{
${ }^{17}$ Blumenberg, Arbeit am Mythos, 10 [Hervorhebung des Vf.].

${ }^{18}$ Kasimir Malewitsch, Die gegenstandslose Welt [1927], Mainz/Berlin 1986, 66.

${ }^{19}$ Dieter Mersch, Nichtpropositionalität und ästhetisches Denken [2013], http:// www.dieter-mersch.de/Texte/PDF-s/ (21.12.2019), 8.
} 
eine solche Synthesis ist es, die das ästhetische Denken performiert. Es ist ein Denken ohne Begriffe, das sich nicht in einer konkreten Aussage zusammenfassen lässt - obwohl es in einem konkreten Gegenstand, in diesem Fall dem schwarzen Quadrat auf weißem Grund, lokalisiert ist und sich autoreflexiv auf dieses selbst richtet, das heißt obwohl es eine Intentionalität besitzt. Diese stellt insofern eine Intentionalität ohne Gegenstand dar, als es sich bei ihr - als Synthesis der De-Synthese - um ein Denken handelt, das auf keinen Begriff kommt.

Auch das Schwarzbild im Film, nicht zuletzt in seinem Oszillieren zwischen Abstraktion und Repräsentation, kann man als Schauplatz einer De-Synthese begreifen, an dem aber doch insofern eine Synthesis geleistet wird, als er der De-Synthese einen Ort, eine Szene gibt. Das Schwarzbild ist in dieser Hinsicht eine Szene, in der Angst im Modus des Ästhetischen gedacht wird.

- Dr. Florian Leitner ist Leiter des Medientheaters am Institut für Musikwissenschaft und Medienwissenschaft der Humboldt-Universität zu Berlin. Seine Forschungsschwerpunkte umfassen die Rolle von Angstaffekten in der Medienkultur, das Verhältnis von Medienperformance und Film sowie Theorien und mediale Ästhetiken der Bewegung. 